\title{
Educação em Saúde nas Doenças Crônico-Degenerativas e a Promoção da Qualidade de Vida: Relato de Experiência ${ }^{1}$ \\ Marta Julia Marques Lopes ${ }^{2}$ \\ Denise Tolfo Silveira ${ }^{3}$ \\ Sandra Rejane Soares Ferreira ${ }^{4}$
}

\section{Resumo}

A experiência relatada advém do projeto de pesquisa-desenvolvimento intitulado: Ambulatório de Promoção da Qualidade de Vida, desenvolvido junto a comunidade da área de abrangência da Unidade Coinma da Divisão de Saúde Comunitária do Grupo Hospitalar Conceição, desenvolvido em integração com a Escola de Enfermagem da UFRGS. A idéia mobilizadora é a de integrar esforços no sentido de adequar a assistência investindo na promoção da qualidade de vida das populações assistidas. A metodologia de intervenção baseia-se no trabalho multidisciplinar e na Consulta de Enfermagem centrada na educação para a saúde. A clientela do ambulatório tem se configurado em sua maioria por pacientes adultos e idosos com problemas crônico-degenerativos e sócio-afetivos.

Palavras-Chave: saúde; qualidade de vida; adultos; idosos; Enfermagem; consulta.

\section{Abstract}

The reported experience emerges from the development-research project named

\footnotetext{
${ }^{1}$ Projeto de pesquisa-desenvolvimento em parceria entre a Escola de Enfermagem/UFRGS e a Unidade Coinma - Divisão de Saúde Comunitária (DSC)/ Grupo Hospitalar Conceição, Porto Alegre, RS.

E-mail:marta@enf.ufrgs.br

${ }^{2}$ Doutora em Sociologia, Paris VII, Professora Titular do Departamento de Assistência e Orientação Profissional, Escola de Enfermagem, Universidade Federal do Rio Grande do Sul (EENF/UFRGS).

${ }^{3}$ Mestre em Assistência de Enfermagem Universidade Federal de Santa Catarina/UFRGS, Professora Assistente do Departamento de Enfermagem Médico Cirúrgica, Escola de Enfermagem, UFRGS.

E-mail: denise@enf.ufrgs.br

${ }^{4}$ Mestranda em Enfermagem EENF/UFRGS, Enfermeira da Divisão de Saúde Comunitária, Grupo Hospitalar Conceição.
}

Estud. interdiscip. envelhec., Porto Alegre, v.2, p.121-130, 1999. 
"Ambulatory of Promotion of Quality of Living", developed alog with community living within the area of Unidade Coinma of de Divisão de Saúde Comunitária of Grupo Hospitalar Conceição, and also developed with the Escola de Enfermagem of Universidade Federal do Rio Grande do Sul. The idea wcich moves the project is to assemble efforts so to adapt assistance, investing in promotion of quality of living for the assisted communities. The methodology is based on the pluridisciplinary work and nursing consultation centred on education for health. The clients of the ambulatory are mostly grown up and aged patients with chronic-degenerative and social-affective problems.

Key-Words: health; quality of living; grown-up; aged people; Nursing consultation.

\section{Introdução}

Em agosto de 1998, um grupo de professoras da Escola de Enfermagem da Universidade Federal do Rio Grande do Sul (EENF/UFRGS), em estágio prático com alunos de graduação na Unidade Coinma da Divisão de Saúde Comunitária - DSC do Grupo Hospitalar Conceição - GHC, propôs-se a, juntamente com a equipe local, investir em uma proposta multidisciplinar de assistência a adultos e idosos com ênfase na educação para a saúde na promoção da qualidade de vida.

Inicialmente, foi elaborado um projeto no qual foi proposta a linha assistencial a ser adotada e a população a ser atendida, ou seja, um investimento em uma mudança de referencial em relação às práticas assistenciais comumente adotadas nos serviços. Foi proposto o atendimento à população de adultos e idosos com problemas crônico - degenerativos. No centro das intenções estava e está a necessidade de adoção da visão integral dos sujeitos atendidos e de um trabalho complementar entre diferentes profissionais, propondo abordagens no plano individual e coletivo.

A equipe foi estruturada com a participação das alunas do curso de graduação, em caráter voluntário e com duas professoras da EENF/UFRGS e a enfermeira do serviço, responsáveis diretas pelo treinamento e supervisão das atividades das alunas. A atividade base da intervenção, no então denominado 
Ambulatório de Promoção da Qualidade de Vida - APQV, foi a Consulta de Enfermagem. Desde então, o ambulatório funcionou com o sistema de agendamento da Unidade Coinma - DSC/GHC, duas vezes na semana, terças e quintas-feiras, atendendo a cinco consultas de 45 minutos em média por tarde.

A equipe coordenadora do projeto foi constituída por duas professoras da EENF/UFRGS, pela enfermeira e um médico residente em Medicina Geral Comunitária do serviço. O grupo executivo ${ }^{5}$ atuante contou com a equipe coordenadora e agregou os demais médicos, auxiliares de enfermagem e auxiliares administrativos da equipe local. Como equipe ampliada, contou, ainda, com um médico psiquiatra do serviço e com uma profissional de educação física que assessorou tecnicamente as atividades junto ao grupo de caminhada e o grupo da $3^{a}$ idade, estruturados na Unidade.

O processo de treinamento das alunas para atuação no ambulatório contou, também, com professores convidados para o desenvolvimento de temas específicos como: envelhecimento, saúde mental e algumas especialidades médicas de interesse para a atenção nos problemas crônico-degenerativos.

\section{A Proposta: caracterização e fundamentos teórico/ metodológicos}

Atualmente, mais que em qualquer outro momento da história, proliferam as pesquisas e os investimentos na área da saúde. $\mathrm{O}$ avanço tecnológico é muito veloz e já permite chegar a conhecimentos, inimagináveis, em outros momentos históricos. Todos esses conhecimentos podem ser facilmente acessados por sistemas de comunicação cada vez mais rápidos, eficientes e variados.

Neste conjunto de descobertas e perspectivas, inseridos na aceleração do saber e por ele impulsionados a levarem uma vida mais agitada e competitiva, estão os indivíduos na fase da vida adulta e idosos, especialmente. $\mathrm{O}$ aumento das exigências no cotidiano profissional e pessoal, o aumento da carga de responsabilidade, a falta de tempo para a atenção a si próprio têm propiciado o

\footnotetext{
${ }^{5}$ Participam da equipe executiva do projeto outros profissionais da Unidade Coinma como o Médico residente em Medicina Geral Comunitária, João José Brum Argoud, e o bolsista de Iniciação Científica (PIBIC/CNPq) João Luís Almeida da Silva, Acadêmico de Enfermagem EENF/UFRGS.
} 
aumento das doenças denominadas de "crônico-degenerativas" como a hipertensão arterial, a diabetes e as doenças relacionadas ao plano sócio-afetivo como ansiedade e depressão.

Analisando essa realidade, foi fortalecida a perspectiva de intervenção em saúde no sentido de que, cuidados com a qualidade da alimentação, a prática de exercícios físicos, o "saber" lidar com o estresse cotidiano, a busca da satisfação na atividade profissional, o aumento de atividades de recreação e lazer, relações familiares e sociais equilibradas e afetivas, entre outros fatores, compõem um conjunto de condições que podem auxiliar indivíduos e grupos na busca de uma vida com qualidade.

Nessa linha de pensamento, passou-se a caracterizar, a perspectiva de intervenção da Divisão de Saúde Comunitária na qual esteve inserida a proposta. Das características do serviço fizeram parte: a atuação em áreas geograficamente delimitadas na cidade e o fácil acesso da população às Unidades; o respeito às práticas e valores culturais da população; a atenção continuada; o registro sistemático das atividades; o desenvolvimento de ações nos diferentes níveis de complexidade - primário, secundário e terciário - e a intervenção no plano individual e no coletivo, bem como o desenvolvimento de ações de promoção, prevenção, cura e reabilitação. Essas características têm assegurado alta resolutividade nas ações desenvolvidas e sistematicamente avaliadas (Giacomazzi et al., 1996).

A Unidade Coinma é uma das treze Unidades de Atenção Primária à Saúde vinculada a DSC. Localiza-se na zona leste de Porto Alegre, na divisa de duas comunidades: o Coinma e a Vila Margarita e foi implantada em maio de 1994.

Segundo o Diagnóstico de Saúde realizado em 1994, a Unidade é responsável por uma população de 4.000 habitantes e atende 920 famílias cadastradas, dois asilos e uma escola de primeiro grau. Reside nas duas comunidades uma população predominantemente de adultos jovens (20 à 59 anos $=53 \%)$; um número significativo de idosos (60 anos ou mais $=11 \%)$ e um pequeno número de menores de um ano (1\%) (Ferreira; Costa, 1994).

Nesse cenário, o projeto teve como objetivo ampliar o atendimento ambulatorial 'a pacientes adultos e idosos, com problemas de saúde comuns 
nessa faixa etária ou com doenças crônico-degenerativas.' Através de Consultas de Enfermagem, prioritariamente, buscou desencadear um processo de educação em saúde visando a promoção da qualidade de vida. As atividades educacionais e assistenciais, da equipe coordenadora, ampliaram as reflexões sobre essa temática auxiliando na construção de uma proposta de consulta de enfermagem específica para a população assistida.

Neste relato é proposta a discussão de alguns aspectos da prática realizada que poderão contribuir na reflexão das práticas e na construção de uma metodologia de intervenção de enfermagem adequada as realidades de saúde, social e cultural das populações assistidas.

\section{A Consulta de Enfermagem e a Promoção da Qualidade de Vida}

Estudos de Horta (1979), referem a importância da Consulta de Enfermagem como uma atividade sistematizada. A sistematização da assistência envolve uma seqüência dinâmica de etapas que direcionam as ações de modo a contribuírem no atendimento às necessidades de saúde do indivíduo e da coletividade. Essa assistência deverá propiciar a identificação de problemas e situações passíveis de resolução à partir de uma atuação conjunta enfermeira/ paciente/equipe multiprofissional ou da efetivação dos encaminhamentos necessários.

Para Adami et al. (1989), a Consulta de Enfermagem contribui com a aplicação dos princípios da universalidade, eqüidade, resolutividade e integralidade das ações de saúde, preconizadas inicialmente pela reforma sanitária e hoje pelo Sistema Único de Saúde - SUS. Segundo a autora, a Consulta de Enfermagem " ( . . . ) visa a avaliação do estado de saúde do indivíduo durante o ciclo vital e o controle de algumas patologias de natureza transmissível e crônico-degenerativas." A consulta possui um cunho educativo, tendo em vista o preparo, tanto do indivíduo como da família, para o autocuidado, em termos de promoção, proteção, recuperação e reabilitação da saúde.

Nessa linha, Ferreira (1996), complementa a idéia quando diz que as consultas de enfermagem tem como objetivo auxiliar na resolução do problema que motivou a consulta, e estabelecer um vínculo terapêutico com a clientela que poderá levar ao inicio de um processo de educação em saúde. Este processo 
visa a promoção do autocuidado e/ou à detecção/prevenção de doenças e/ou dos riscos para problemas de saúde, em função da faixa etária, da ocupação/ trabalho, do ambiente/sociedade em que se vive.

Nessa perspectiva, a proposta metodológico-assistencial e as consultas em particular, foram se desenvolvendo sem uma abordagem abrangente e dinâmica, buscado uma visão multidimensional dos indivíduos, famílias e comunidades no sentido de entender seu processo de interação com fatores sócio-ambientais que compõem o processo saúde-adoecimento ou que servem de estímulo ao processo de autocuidado.

A Consulta de Enfermagem tem como base as seguintes questões: como ajudar as pessoas? Como a relação de consulta pode ser competente para os dois lados?

Para que tais questões sejam respondidas, foi necessário a adequação da conduta enquanto consultores centrados em nós mesmos, pois, freqüentemente, há um esforço em responder às nossas próprias necessidades e não as dos pacientes. Geralmente há uma preocupação mais com o racional do que com o emocional.

Examinando essa conduta dominante nas nossas atitudes de consultório e nas práticas em serviços comunitários, tentou-se criticamente, adotar perspectivas inovadoras. Assim, houve um esforço em adotar as atitudes a seguir descritas:

- a escuta ativa, que se constitui em um engajamento no sentido de estar "com você" - tentando desenvolver no outro o sentimento de "ser compreendido";

- a aceitação do outro, além do que se pensa ou do que ele diz, o que nos permite desenvolver essa aceitação compreensiva e não normativa;

- a empatia, que se constitui em um esforço em compreender os sentimentos das outras pessoas e não apenas suas idéias;

- a confiança, que é construída no desenrolar da relação de consulta e se desenvolve a partir de um tomada de consciência das próprias emoções do profissional e do manejo adequado na direção da ação terapêutica; 
- a ausência de julgamento, que pode se constituir em uma forte aliada no sentido de que julgar negativamente uma conduta do paciente nem sempre resulta no desencadeamento de uma atitude adequada e de adesão à terapêutica (Lopes, 1999).

Do ponto de vista técnico institucional, as Consultas de Enfermagem do ambulatório, obedeceram às normas básicas e à literatura específica de enfermagem no campo assistencial. Dentre as normas e os suportes legais, devem ser citados: a Legislação do Exercício Profissional de Enfermagem (Lei no 7.498/86 e Decreto n 94.406/87); a Resolução no 195 do COFEN Conselho Federal de Enfermagem (dispõe sobre a solicitação de exames laboratoriais); e as normas protocolares estabelecidas nesse âmbito pela Divisão de Saúde Comunitária do Grupo Hospitalar Conceição (DSC/GHC) (Devinar; Ferreira, 1994; Ferreira, 1993).

A primeira etapa da consulta esteve mais voltada para a construção da história pessoal e de vida do paciente. Portanto, utilizou-se a coleta de dados, a pesquisa dos problemas e/ou fatores nocivos para, posteriormente, desenvolver e fundamentar a proposta terapêutica. Na primeira consulta, nem sempre é necessário realizar o exame físico completo ${ }^{6}$, ele pode ser iniciado com base nas queixas principais do paciente e ser concluído em uma consulta subseqüente. Para que se possa ter uma avaliação das condições gerais de um indivíduo adulto, por exemplo, recomendou-se alguns dados essenciais, tais como: seu estado geral, condições de vestuário, condições mentais, expressão facial, condições de locomoção, peso, altura, freqüência e ausculta cardíaca, frequiência e ausculta respiratória e valores de temperatura corporal e tensão arterial.

Foi proposta a prestação de um atendimento em equipe multiprofissional e com visão do paciente como um todo; portanto, quanto mais amplas fossem as capacitações para diagnosticar situações de saúde-adoecimento, mais abrangente poderia ser a atuação da equipe. Quando identificados problemas

\footnotetext{
${ }^{6} \mathrm{O}$ exame físico é um componente indispensável da consulta de enfermagem e inclui as seguintes técnicas: inspeção, palpação, percussão e ausculta. Destina-se a identificar evidência física de capacidade funcional e/ou incapacidade funcional apresentada pela clientela atendida. Ele requer distinção exata de sinais normais e anormais, como também variações do normal entre os clientes e os graus de normalidade num mesmo cliente (Adam et al., 1989).
} 
fora da competência do grupo, o achado foi registrado e encaminhado para o profissional adequado.

O plano assistencial teve por objetivo estabelecer prioridades aos problemas e/ou fatores nocivos (estressores) identificados, especificar os resultados que se espera que o paciente alcance e designar as ações especificas de intervenção, e deve ser traçado junto com o paciente. Acredita-se que durante o processo de interação, algumas vezes, será necessário negociar e/ou fazer contratos para uma efetiva adesão à terapêutica. Ou seja, é preciso procurar, ao longo da consulta, desenvolver uma aliança terapêutica, ir completando (relembrando) as questões fechadas anteriormente para seguir adiante ou retomar a discussão. No fechamento da consulta é essencial combinar a periodicidade necessária para os retornos.

Os registros das etapas de todo o processo descrito, foram realizados no instrumento (protocolo) de sistematização da consulta e no prontuário do paciente (prontuário de família) utilizado pelo serviço. A evolução de enfermagem, compreendeu o registro das respostas dos pacientes aos cuidados planejados, ao programa terapêutico posto em prática e às orientações e informações recebidas, bem como, de novos dados manifestados pelo paciente ou observados pela enfermeira.

Adotando essas perspectivas foi proposta a realização de consultas de forma integrada e complementar, construindo uma perspectiva comum interprofissionais para o atendimento da clientela.

\section{As Atividades Integradas}

As atividades integradas correspondem à complementaridade no momento da consulta, entre auxiliares, enfermeiras e médicos da Unidade; aos encaminhamentos interprofissionais dando continuidade as propostas terapêuticas para cada paciente, individualmente. Pode-se citar, como exemplo, a proposta assistencial para pacientes com HAS (Hipertensão Arterial Sistêmica) os quais necessitam terapia medicamentosa além de um planejamento do ponto de vista das atividades físicas, do controle alimentar, do estresse, entre outros. Cada profissional registrou sua avaliação e a conduta adotada. Uma atitude constante foi a de investir o tempo da consulta na ação educativa, visando a 
adequação do comportamento através da participação ativa do indivíduo na sua recuperação, como sujeito responsável pela sua saúde e qualidade de vida.

Outra atividade integrada e continuada envolveu a educação para a saúde a grupos e a equipe desenvolveu uma atividade mensal denominada Conversando sobre ..., a qual que se propõe a atender as necessidades de informação e discussão coletiva de algumas temáticas prevalentes no ambulatório. Os temas trabalhados foram definidos a partir de sugestões dos grupos entre eles o da $3^{\text {a }}$ Idade e pela problemática epidemiológica, advinda das situações trazidas às consultas. Até o momento, foram trabalhados temas como: osteoporose; alimentação, saúde e qualidade de vida; climatério; atividade física.

\section{Considerações Finais}

Pensa-se que a equipe multiprofissional, trabalhando com uma intenção interdisciplinar, pode iniciar um processo de rompimento com as práticas compartimentadas, fragmentadas, dissociadas da realidade social e política, presentes ainda na realidade dos serviços de saúde. Trabalhando com essa concepção pode-se romper com as práticas lineares que predominam no modelo biologicista de assistência à saúde, buscando práticas que considerem a estrutura social, na qual, os indivíduos e grupos estão inseridos, tornando-as mais abrangentes, resolutivas, integradoras, com qualidade e em quantidade suficiente para atender as demandas da população.

Por fim, enquanto grupo envolvido na proposta, acredita-se que as atividades não se esgotam na assistência, sendo necessário ampliá-las no campo do ensino e da pesquisa, no sentido de qualificar e aprofundar as abordagens do processo saúde-adoecimento na perspectiva da qualidade de vida.

\section{Referências Bibliográficas}

1 ADAMI, Nilce P. et al. Características Básicas que Diferenciam a Consulta de Enfermagem da Consulta Médica. Acta Paulista de Enfermagem, São Paulo, v. 2, n 1, p. 9-13, mar. de 1989. 
2 DEVINAR, L. A.; FERREIRA, S. R. S. (Org.). Serviço de Saúde Comunitária.Rotinas de Solicitação de Exames Laboratoriais para Enfermeiras do DSC/GHC. Porto Alegre, mar. de 1994, mimeogr.

3 FERRAZ, S. T. A Pertinência da Adoção da Filosofia de Cidades Saudáveis no Brasil. Saúde em Debate, Londrina. n. 41, p.9-13, dez. 1993.

4 FERREIRA, S. R. S.(Org.). Rotinas de Prescrição, Transcrição de Medicamentos e Procedimentos para Enfermeiras dos Ambulatórios do HNSC e DSC/GHC. Porto Alegre, Grupo Hospitalar Conceição, Divisão de Saúde Comunitária, Serviço de Saúde Comunitária, mar. 1993. mimeogr.

5 FERREIRA, S. R. S. et al. História e Avanços no Trabalho de Enfermagem na Divisão de Saúde Comunitária do Grupo Hospitalar Conceição. Momento \& Perspectivas em Saúde, Porto Alegre. v.9, n. 2, p.13-18, jul/dez.1996.

6 FERREIRA, S. R. S.; COSTA, M. (Org.). Relatório do Diagnóstico de Saúde das Comunidades Coinma e Vila Margarita. Unidade Coinma, Porto Alegre, 1994, mimeogr.

7 GIACOMAZZI, A. (Org.). Apresentação. Momento \& Perspectivas em Saúde, Porto Alegre. v.9, n. 02, p.7, jul./dez. 1996.

8 HORTA, W. A. Processo de Enfermagem. São Paulo, EPU, Ed. da Universidade de São Paulo, 1979.

9 LEE, J. O Projeto de Cidades Saudáveis. Conferência do Seminário sobre o Projeto de Cidades Saudáveis. Toronto, 1994.

10 LEMOS, C; \& ERTHAL, C. I. Cartilha da Longevidade. Porto Alegre, Nova Era, Grupo de Educação em saúde, Série 1, Fasc 2, 1998.

11 LOPES, M. J. M. A Consulta de Enfermagem: alguns pressupostos. Porto Alegre, EENF/UFRGS, 1999. mimeogr.

12 SILVEIRA, D. T. Consulta-ação: educação e reflexão nas intervenções de enfermagem no processo trabalho-saúde-adoecimento. Porto Alegre, UFRGS/UFSC, 1997. Dissertação de Mestrado. 